\title{
A meta-analysis on major risk factors of multiple primary cutaneous melanomas
}

\author{
Vivien Marasigan ${ }^{1}$ \\ Eric Schmitt ${ }^{2}$ \\ Marjan Garmyn ${ }^{3}$ \\ Wim Van den Noortgate ${ }^{4}$ \\ 1 University Hospitals Leuven, Belgium \\ 2 Department of Mathematics, Catholic University \\ Leuven, Belgium \\ ${ }^{3}$ Department of Dermatology, University Hospitals \\ Leuven, Belgium \\ ${ }^{4}$ Leuven Statistics Center, Catholic University Leu- \\ ven, Belgium
}

Address for correspondence:

Vivien Marasigan, MD

University Hospitals Leuven

Belgium

E-mail: vivien.marasigan@uzleuven.be

\section{Summary}

Background. Identifying multiple primary melanoma (MPM) risk factors is an important step towards detecting secondary primaries at an early stage and optimizing follow-up of melanoma patients. Therefore, we examined the risk factors for MPM versus single primary melanomas (SPM) in a meta-analysis.

Methods. Published literature comparing risk factors for MPM versus SPM up until May 13, 2013 were retrieved from Embase and MEDLINE databases. Effect sizes were aggregated and analyzed using random effects models (REM) using Bayesian methods.

Results. 16 articles comparing risk factors between MPM versus SPM, comprising of 2442 MPM and 24.895 SPM individuals were included. Positive family history (OR 2.0,95\% HPD:1.6-2.7), presence of dysplastic nevi (OR 4.4,95\% HPD: 2.6-8.9) and light hair color (OR 1.4,95\% HPD:1.1-1.7) were shown to be significantly linked with MPM. Bayesian estimates of other factors, including benign nevi count, sunburn history, freckles and phototype were not significantly raised.

Limitations. Heterogeneity between studies and the small number of eligible papers limited our meta-analysis.

Conclusion. This meta-analysis confirms that a positive family history, presence of dysplastic nevi and light hair color correlate with higher risk for developing MPM. Patients with these characteristics should be carefully monitored.

KEY WORDS: melanoma; multiple primaries; metaanalysis; epidemiology; secondary prevention; risk factors.

\section{Introduction}

Cutaneous malignant melanoma is the most frequent cause of skin cancer related death. With the incidence increasing steadily (1), understanding its etiology is a pressing issue. Major risk factors associated with development of melanoma are: high common naevi count, presence of dysplastic naevi (DN), a positive family history, actinic damage, fair skintype, intermittent sun exposure and sunburn history (2-4). Although most patients do not develop more than one melanoma, a first melanoma is positively correlated with developing a second melanoma, with percentages ranging from 0.2 to $20 \%(5-7)$. However, the incidence of multiple primary melanomas (MPM) is relatively low in the general population, making it difficult to study. Begg et al. (8) proposed a solution to this problem by comparing multiple primaries (cases) to single primaries (controls), thereby increasing statistical efficiency. Knowledge of which risk factors is more prevalent in multiple primary melanoma patients compared to single primary melanomas (SPM) patients might not only be useful for secondary prevention but might also lead to greater understanding of the etiology of multiple primary melanoma.

For this purpose, we investigated the risk factors associated with multiple primary melanomas versus single primary melanomas through a meta-analysis.

\section{Methods}

\section{Search strategy}

Relevant literature in the Embase and MEDLINE (using PubMed) databases published from inception through May 13, 2013 was investigated with the aid of experienced librarians. The following keywords were used for Pubmed: (Secondary melanoma* OR second melanoma* OR multiple melanoma OR multiple melanomas OR mulitiple primary melanoma*) AND ("Risk Factors"[Mesh] OR "Risk Assessment"[Mesh] OR risk OR risks OR predictors OR "Sunlight"[Mesh] OR "Sunburn"[Mesh] OR sunburn* OR sunlight OR Sunshine OR "sun exposure" OR "Keratosis, Actinic"[Mesh] OR actinic keratos* OR ((atypical OR dys- 
plastic OR common) AND ("Nevus"[Mesh] OR naevi OR nevi)) OR "family history" OR "Phenotype"[Mesh] OR Phenotype*[tiab] OR "solar lentigo" OR "solar lentigines”). For EMBASE: secondary NEAR/2 melanoma OR second NEAR/2 melanoma OR multiple NEAR/2 melanoma. The results were entered in Endnoteweb to facilitate an overview and to detect redundant articles. Relevant articles from these sources and reference lists were chosen based on titles and abstracts. The Meta-analysis of Observational Studies in Epidemiology (MOOSE) guidelines were used to guide the analysis (9).

Included studies were: human observational and epidemiological studies in adults comparing clinical risk factors for cutaneous MPM versus SPM for which full length original research articles are available and which contain enough information for an estimation of an effect size and standard error.

Excluded studies were: non-human studies, reviews, case reports, editorials, or studies that focus only on specific types of melanoma or certain subgroups.

The following information was collected from each study: bibliographical information, country of the study, study population, study design, type of effect size, definition of the risk factor, and quantitative data for effect size calculation. If two or more studies were based on the same/overlapping samples, the one with the largest sample or best definition of the risk factor and the estimates for a risk factor that were most adjusted by controlling for other covariates were used. Effect size estimates that were not explicitly available were calculated from information that was provided.

Articles published in languages other than English were included provided that the title or the abstract was in English so that they could be detected. In the second step, only papers comparing melanoma risk factors in a multiple melanoma population against a single melanoma population were included.

Two reviewers independently extracted data from all included studies. Disagreements were resolved through discussions and arbitration of a third reviewer.

\section{Statistical Methods}

Most papers reviewed presented odds ratios (ORs), so we focused on presenting results for this metric. ORs and corresponding confidence intervals $(\mathrm{Cl})$ were retrieved, or calculated from summary or crude data. Subsequently, the natural logarithm of the ORs (log OR) and sampling variances were calculated.

Bayesian estimates of the random effects model (REM) parameters were derived using the Openbugs software (10). REMs have the advantage that they can account for heterogeneity between studies when estimating the size of the population effect. In contrast to maximum likelihood estimation (MLE), Bayesian estimation techniques do not rely on asymptotic assumptions, making them more suitable for small samples. We select priors that strongly assume there is no significant effect for the random effects. In doing so, we estimated models that are unlikely to find significance if the sample is small and heterogeneous. Such a prior is known as a skeptical prior. This choice was made partly as a way to avoid spurious results given the small sample sizes of many of the risk factors as suggested by Duminda et al. (11). Although incorrectly identifying a risk factor as significant might be preferable to ignoring an important one, a goal of this meta-analysis is to draw attention to risk factors that require further study. On the other hand, in spite of the small samples, if our meta-analysis finds a risk factor significant, it is likely that this factor genuinely affects the risk. Typically, for our specification, as the sample size grows, the Bayesian estimates quickly resemble those estimated using MLE.

This modeling approach produces estimates of a posterior density function for the mean log OR and for $\mathrm{tau}^{2}$. A Bayesian alternative to a confidence interval is to consider highest posterior density interval (HPD). Log ORs are converted to ORs and the risk factor is considered significant if 1 is not included in the interval. To summarize the studies considered for the risk factors, we present the corresponding ORs and their confidence intervals in forest plots (12).

For family history, a relatively high number of studies were available in contrast to other risk factors, therefore heterogeneity between studies was investigated by estimating and testing the estimated between study variance, $\mathrm{tau}^{2}$, from the REM. Based on these results, the results of similar analyses for smaller sample sizes are not discussed.

\section{Results}

Our initial broad search found 2993 articles in Embase and 1681 in MEDLINE, totaling 4674 articles (Figure 1). After deduplication, 3576 articles remained. Evaluation through scrutiny of titles and abstracts rendered 130 potential articles. A manual study of reference lists yielded an additional 20 articles. The inclusion/ exclusion criteria was further explored whereby 18 articles were identified. Marghoob et al. (13) presented cases with classic atypical mole syndrome only, and therefore cannot be compared with other papers. Siskind et al. (7) reported hazard ratios instead of ORs and contained no crude data. These two studies were excluded. In total, 16 studies had data on at least one of the aforementioned risk factors (Table 1).

\section{Family history}

Twelve studies were included in the analysis (Table 1). Of these, five studies did not give a precise definition of a positive family history, but were specific enough to remain usable $(8,14-17)$. Five studies defined family history $(\mathrm{FH})$ to be positive when at least one first-degree family member has a history of melanoma $(5,18$ 21) whilst two studies (22-23) determined $\mathrm{FH}$ to be at least one first or second-degree family member with a melanoma. Four studies provided ORs $(8,15,18,23)$. One of these studies utilized logistic regression analysis, which produces log ORs as results, which the authors identified by the term relative risk (18). The OR was calculated from crude data in eight studies $(5,14$, 16, 17, 19-22). 


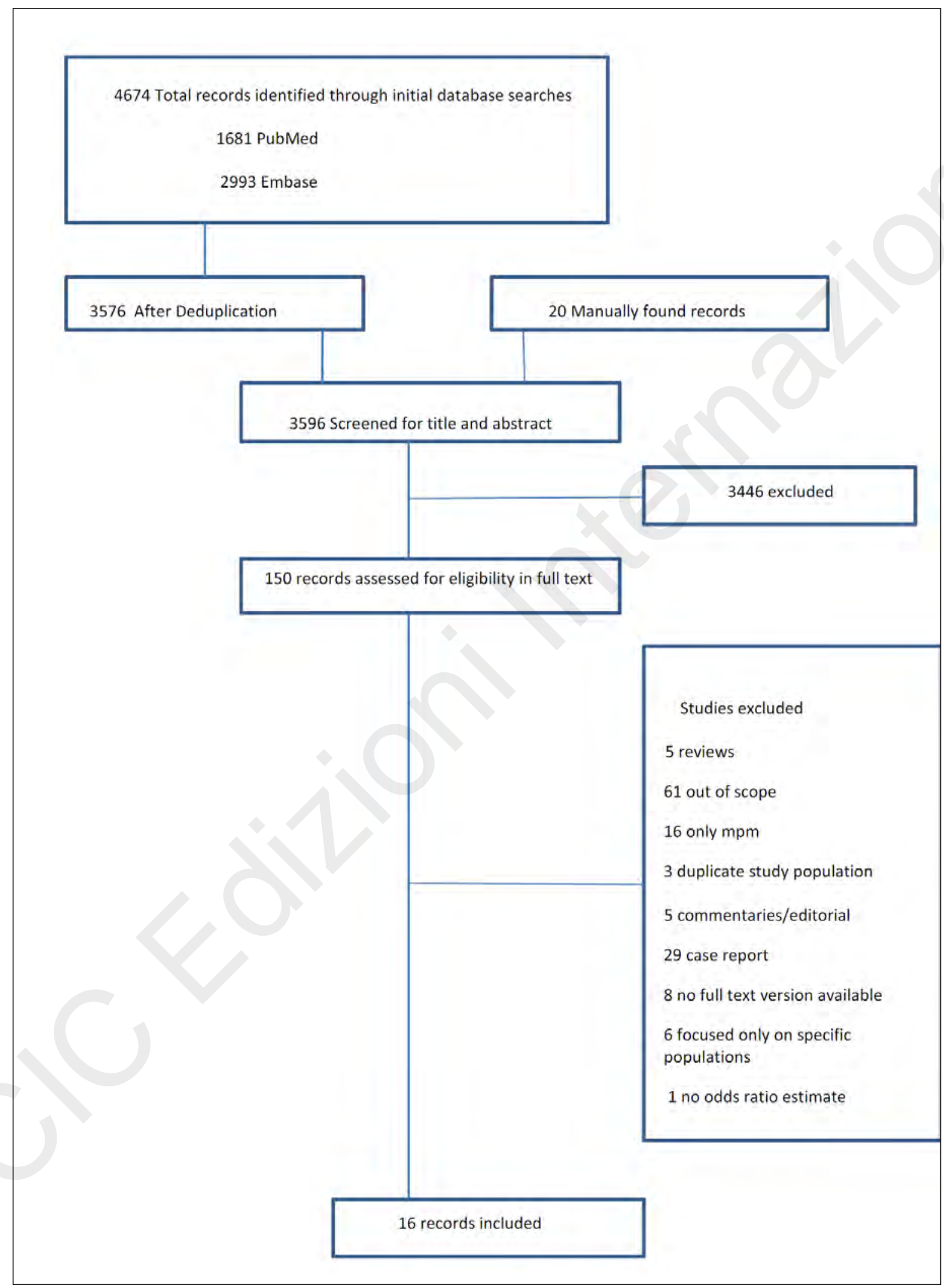

Figure 1 - Flow diagram of article selection process. 
Table 1- This table depicts characteristics of the studies used for the meta-analysis for a certain risk factor.

\begin{tabular}{|c|c|c|c|c|c|c|c|c|c|c|c|c|}
\hline STUDY & YEAR & COUNTRY & MPM $^{1}$ & $\mathrm{SPM}^{2}$ & $\mathrm{FH}^{3}$ & $\mathrm{DN}^{4}$ & $\mathrm{BN}^{5}$ & $\mathrm{HC}^{6}$ & $\mathrm{SB}^{7}$ & $\mathrm{FR}^{8}$ & $\mathrm{EC}^{9}$ & $\mathrm{PT}^{10}$ \\
\hline $\begin{array}{l}\text { Ferrone } \\
\text { et al. (5) }\end{array}$ & 2005 & USA & 385 & 4484 & $x$ & $x$ & & & & & & \\
\hline Begg et al. (8) & 2006 & Int'| GEM & 1210 & 2470 & $x$ & & $x$ & $x$ & & $x$ & $x$ & $x$ \\
\hline $\begin{array}{l}\text { Scheibner } \\
\text { et al. (14) }\end{array}$ & 1982 & Australia & 90 & 3128 & $x$ & $x$ & & & & & & \\
\hline $\begin{array}{l}\text { de Giorgi } \\
\text { et al. (15) }\end{array}$ & 2010 & Italy & 40 & 672 & $x$ & $x$ & $x$ & & & & & $x$ \\
\hline $\begin{array}{l}\text { Hwa } \\
\text { et al. (16) }\end{array}$ & 2012 & USA & 61 & 727 & $x$ & & & & & & & \\
\hline $\begin{array}{l}\text { Pastorino } \\
\text { et al. (17) }\end{array}$ & 2008 & Italy & 95 & 398 & $x$ & & & $x$ & & $x$ & $x$ & \\
\hline $\begin{array}{l}\text { Burden } \\
\text { et al. (18) }\end{array}$ & 1999 & U.K. & 48 & 108 & $x$ & $x$ & $x$ & & & & & \\
\hline $\begin{array}{l}\text { Sigg } \\
\text { et al. (19) }\end{array}$ & 1989 & Switzerland & 23 & 257 & $x$ & & & & & & & \\
\hline $\begin{array}{l}\text { Manganoni } \\
\text { et al. }(20)\end{array}$ & 2012 & Italy & 76 & 2079 & $x$ & & & & & & & \\
\hline $\begin{array}{l}\text { Nagore } \\
\text { et al. (21) }\end{array}$ & 2008 & Spain & 36 & 923 & $x$ & & & & & & & \\
\hline $\begin{array}{l}\text { Slingluff } \\
\text { et al. (22) }\end{array}$ & 1993 & USA & 283 & 7816 & $x$ & & & & & & & \\
\hline $\begin{array}{l}\text { Titus } \\
\text { Ernsthoff } \\
\text { et al. (23) }\end{array}$ & 2006 & USA & 27 & 327 & $x$ & $x$ & $x$ & $x$ & $x$ & $x$ & $x$ & $x$ \\
\hline $\begin{array}{l}\text { Titus } \\
\text { Ernsthoff } \\
\text { et al. (24) }\end{array}$ & 1988 & USA & 8 & 24 & & $x$ & $x$ & $x$ & $x$ & & & $x$ \\
\hline $\begin{array}{l}\text { Mandelcorn } \\
\text { et al. (25) }\end{array}$ & 2011 & Int'I GEM & 1138 & 2151 & & & & & & & & $x$ \\
\hline $\begin{array}{l}\text { Johnson } \\
\text { et al. (26) }\end{array}$ & 1998 & USA & 60 & 1482 & & & & $x$ & & & $x$ & $x$ \\
\hline $\begin{array}{l}\text { Kricker } \\
\text { et al. (27) }\end{array}$ & 2007 & Int'I GEM & 1125 & 2023 & & & & & $x$ & & & \\
\hline
\end{tabular}

${ }^{1}$ MPM: number of participating patients with multiple primary melanomas

2 SPM: number of participating patients with single primary melanomas

${ }^{3} \mathrm{FH}=$ family history

${ }^{4} \mathrm{DN}=$ dysplastic naevi

${ }^{5} \mathrm{BN}=$ benign naevi

${ }^{6} \mathrm{HC}=$ hair color

$7 \mathrm{SB}=$ sunburn

${ }^{8} \mathrm{FR}=$ freckles

${ }^{9} \mathrm{EC}=$ eyecolor

$10 \mathrm{PT}=$ phototype 
All estimates were transformed into log OR and sampling variance. An inverse-variance weighted metaanalysis was performed to obtain an estimate of the overall log OR, which was back-transformed to the OR scale to ease the interpretation. The overall OR was significant OR 2.0 (95\% HPD:1.5-2.7), which means that the odds for a positive family history is twice as large in the MPM group compared to the SPM group. Substantial heterogeneity between studies was not found to be present (estimated $\operatorname{tau}^{2}=0.107,95 \%$ HPD: 0.0-0.5). However, this is likely due to the small sample size and our selection of a very small value for the prior for the heterogeneity parameter. Figure 2 shows a forest plot of these results.

A sub-analysis of the 5 studies which defined a family history as positive only when melanoma occurred in first-degree family members, produced an OR 2.50 (95\% HPD:1.6-5).

\section{Naevi}

In total five studies on common naevi were deemed eligible. Three studies defined common naevi count dichotomously with varying cut-off points $(18,23,24)$. One study had five ordinal groups whereby the estimate of the highest level was included in the analysis (8) whilst one study analyzed common naevi count as a continuous variable (15). Benign nevi was counted for the whole body in three studies $(8,23,24)$ but was not defined in two $(15,18)$. All estimates were transformed into log OR and a Bayesian random effects meta-analysis was applied, obtaining an OR of 1.8 (95\% HPD:0.8-4.5) which is not significant.
Odds ratios could be retrieved from six studies on $d y s$ plastic naevi. Concerning the definition of DN (clinical versus histological), three studies observed DN clinically $(14,15,23)$ whilst two included both clinical DN and histologically confirmed $\mathrm{DN}(5,24)$. One study presented separate results for clinical and histological DN (18). Concerning the quantity of DN: three studies compared the presence of $\geq 1$ DN vs none $(5,15,18)$. One study had two groups for clinical DN: group 1 (>10 vs $2-9$ vs 1 vs 0 ) and group 2, ( $>2$ vs <2) whilst histological DN was categorized as 0 vs rest (24). Another study used 3 categories ( $\geq 3$ vs 1-2 vs 0 ) (23). Scheibner et al. (14) compared $>20$ DN versus $<20$. Using log ORs from the analyses most accounting for covariates adjusted analyses performed by each of the papers, we obtain an aggregated OR of 4.4 (95\% HPD:2.7-7), showing that there is a significant risk of multiple melanoma if at least one DN is observed (Figure 3). A sub-analysis without the Scheibner et al. paper produced an OR of 3.2 (95\% HPD:2.2-6.1), which further bolsters our findings.

\section{Phenotypical features}

Six studies on phototype were examined of which three compared phototype higher than 2 (darker skin) to phototype 1-2 (fair skin) $(15,25,26)$. Begg et al. (8) measured tannability but was in the same collaboration group as Mandelcorn et al. (25) which mentioned phototype; a more precise definition. The latter article was therefore included for this risk factor. Both papers by Titus-Ernstoff et al. $(23,24)$ with two different populations mentioned tannability. The first paper had on-

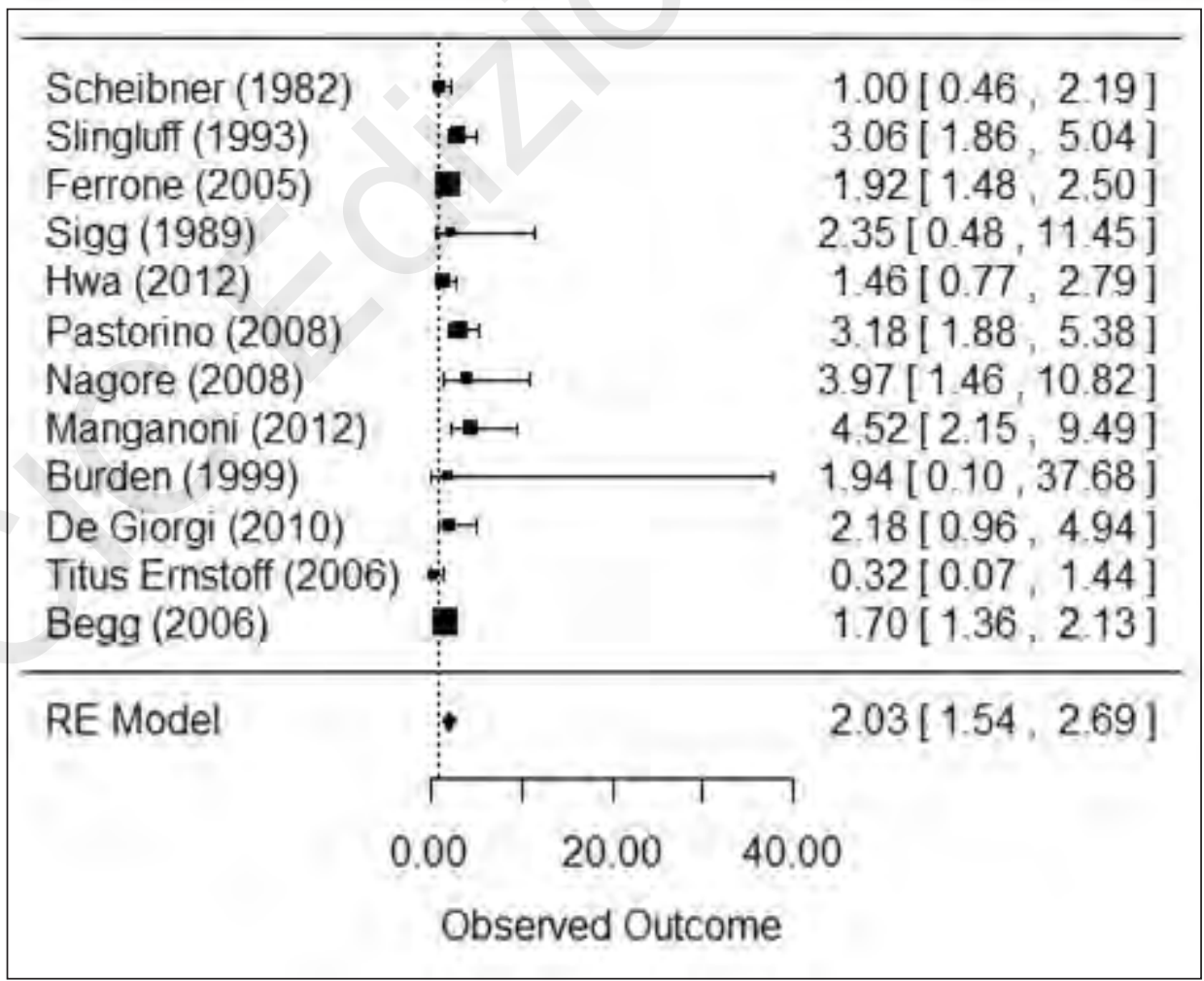

Figure 2 - Forest plot of the ORs and confidence intervals of studies on family history of melanoma. 


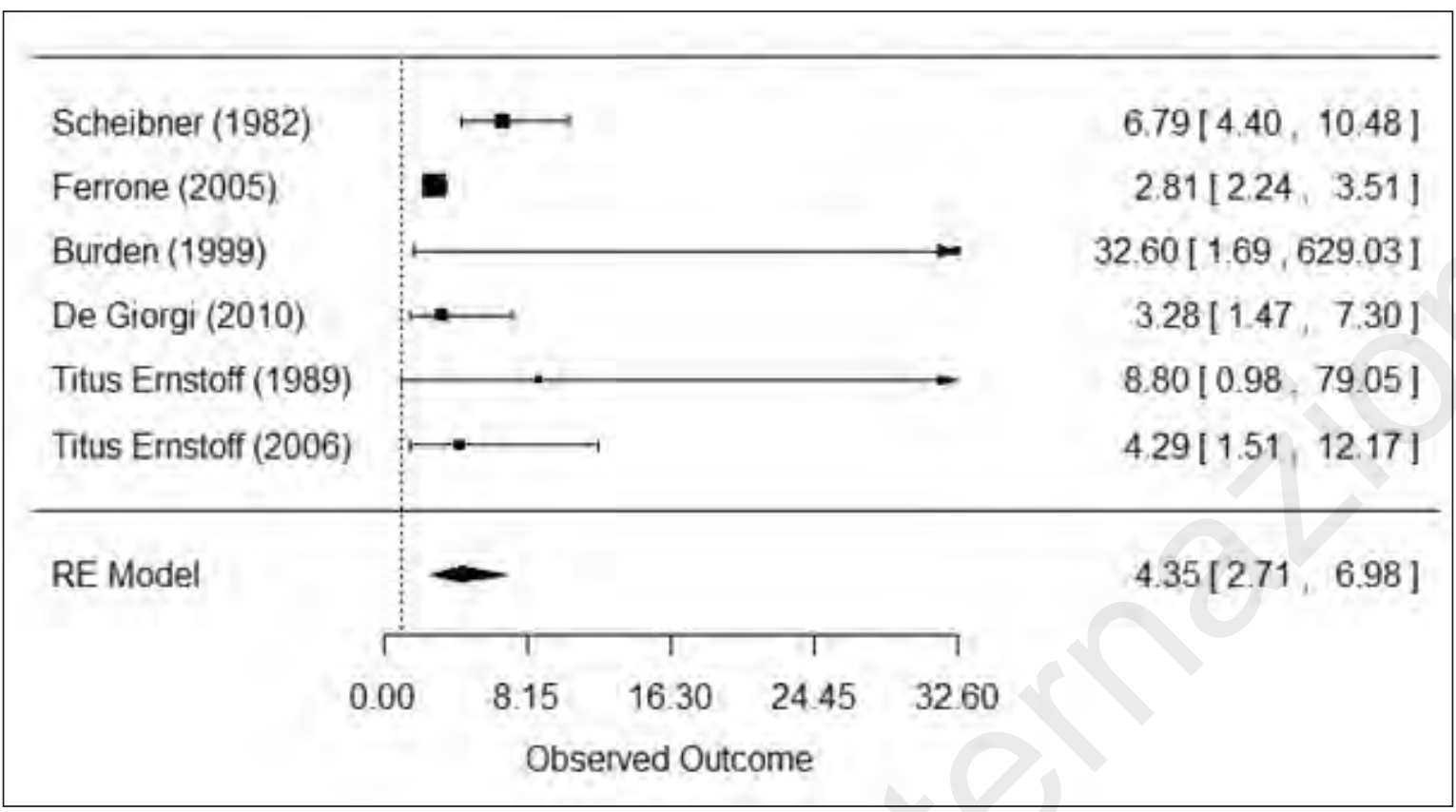

Figure 3 - Forest plot of the ORs and confidence intervals of studies on dysplastic naevi.

ly one estimate (tanning vs no tanning) whereas the latter article published two results: tannability after short term sun exposure vs long term. For the latter study, tannability after long term exposure was chosen because of its higher dependability.

Using the category fair vs dark, gives an OR 1.36 (95\% HPD:0.93-2.01) which is not significant.

There were five studies on hair color included. TitusErnstoff et al. compared red/light hair vs dark in their first publication (24) but mentioned three categories in their second (23) (blonde/red vs brown/redbrown vs black/brown). Begg et al. (8) had estimates for a dichotomous categorization (light $v s$ dark) and for 4 groups (red vs blonde vs light brown vs black/darkbrown). Johnson et al. (26) and Pastorino et al. (17) each had 4 and 3 groups respectively whereby ORs were calculated from crude data (non-dark hair vs dark hair). For this analysis, individuals with the lightest hair color classification were compared to all individuals with darker hair classifications resulting in a dichotomization of the groups (light vs dark hair).

The mean effect estimate was significant: OR 1.4 (95\% HPD:1.2-1.6) (Figure 4).

Three studies mentioning freckles were found, with all three defining freckles as present vs not present. The pooled REM effects were insignificant OR 1.1 (95\% HPD:0.5-1.7), but the sample size is small. Therefore, we performed a sensitivity analysis with fixed effects model, OR $1.2(95 \% \mathrm{Cl} 1.02-1.45)$, which is significant. We also examined four studies mentioning eye color. Begg et al. (8) had two estimates of which the one for light $v s$ dark was used. For the 3 studies without an estimate for a dichotomous variable (light vs dark) $(17,23,26)$, ORs were calculated from the available crude data (non-brown color vs brown). For eye color, the pooled REM effects were insignificant OR
1.0 (95\% HPD:0.7-1.6). Due to the small sample size, a FEM was estimated, also finding insignificance OR 1.1 (95\% Cl: 0.7-1.7). Only two studies $(17,23)$ mentioned skin color with two different ascertainment methods and were therefore not included in the metaanalysis.

\section{Sun exposure}

Three studies on sun burn were included. Two studies $(23,24)$ had dichotomous categories (no sun burn versus one or more episodes). An additional study (27) had multiple ordinal categories of which the highest level (versus no sunburn) was included in the analysis. Using a random effects model, the mean effect estimate was OR 1.1 (95\% HPD:0.1-7.2). However, sensitivity analysis using a fixed effects model found OR 1.4 (95\% Cl 1.1-1.8). In addition to sun burn, three studies on intermittent sun exposure were found but two were from the same collaboration study (GEM). We finally decided not to include the papers in this meta-analysis because of the insufficient number. Articles on actinic damage were sought but only two articles $(14,27)$ were deemed eligible with two completely different sets of variables analytic methods; therefore a meta-analysis was not conducted.

\section{Discussion}

In this meta-analysis we examined whether certain melanoma risk factors are positively associated with the development of a second melanoma. We investigated the following major melanoma risk factors: family history, benign naevi count, dysplastic naevi, phenotypic features (hair color, freckles, eye color) and sun exposure. 


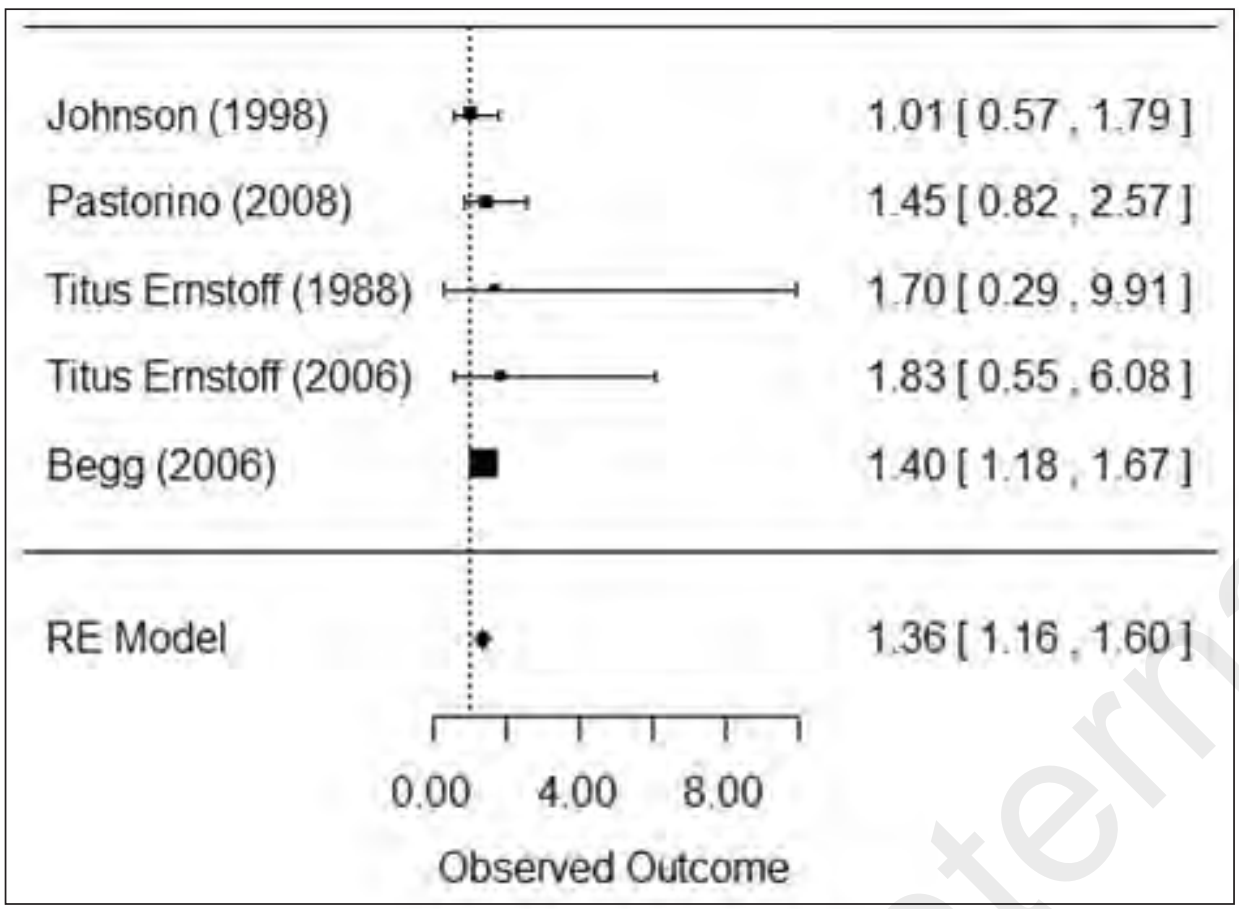

Figure 4 - Forest plot of the ORs of studies hair color.

We only included papers which compared major melanoma risk factors in a multiple melanoma population against a population with a single melanoma only. There are papers which either investigated risk factors within a multiple melanoma population whilst other papers compared multiple melanoma groups with healthy controls. However, because some melanoma risk factors have relatively low prevalence in healthy, population-based controls, such as family history of melanoma and dysplastic naevi, Begg et al. (8) proposed the utilization of single melanoma populations as controls to increase the power of the study.

The major findings of this study were that family history and dysplastic nevi are significantly positively associated with multiple melanoma when compared to a single melanoma population; with dysplastic naevi displaying a much stronger association. These findings concur with the results of meta-analyses on risk factors for melanoma development in the healthy population $(2,4)$

Most studies focused on family history, which was rather unsurprising because genetic susceptibility is known to play a major risk for melanoma development. About $10 \%$ of melanoma cases are familial with high penetrant genes such as CDKN2A (on chromosome 9p21.3) and CDK4 (on chromosome 12q14) accounting for melanoma risk in $20-57 \%$ of familial melanoma, but a very low frequency in sporadic cases (28-30). This implies that other susceptibility genes exist for melanoma susceptibility like low penetrant genes.

The presence of dysplastic naevi is a risk factor associated with the highest risk of acquiring multiple melanoma. The presence of even one dysplastic nae- vi is found to indicate risk. However, most studies that were included categorized dysplastic naevi dichotomously and only one study had ordinal categories for this risk factor which makes a sub-analysis at the level of ordinal groups impossible. Conversely, CDK6 and XRCC1 variants were shown to be associated with DN risk but not with melanoma development in melanoma families implying that other genetic or extrinsic factors (e.g. sun protection usage in risk individuals, solar exposure) might influence the transformation of DN into melanoma (31).

Our findings on light hair color versus dark indicate a significant positive association with multiple melanoma, albeit to a lesser extent. However, the number of papers on phenotypic features is generally low. The estimate for freckles in REM was insignificant in contrast to that of FEM but given the small sample size and the insignificant estimate in the conservative REM, we consider this sensitivity analysis an indication that further studies are needed, rather than conclusive. Findings on phototype and eye color were not significant.

It is evident that studies on sun exposure and indicators of actinic damage in relation to multiple melanoma versus single melanoma populations are lacking. Only sunburn had enough studies to carry out a meta-analysis, but even then there were only three studies. Nevertheless, summary estimates for sunburn were non-significant in the Bayesian analysis but significant in the FEM model. The interpretation of these contradictory results is delicate because the Bayesian approach used for the REM is inherently skeptical, and the sample size is small. The estimates for intermittent sun exposure suggests a positive influence but this is based only on two papers $(14,27)$ (re- 
sults not shown). This result indicates that further research on this risk factor is advisable.

In meta-analyses, heterogeneity between papers can be a limitation when interpreting the results. To conduct our analyses, we estimated random effects models, to account for heterogeneity between papers, using a Bayesian procedure. Due to the small sample sizes for many of the risk factors, Bayesian estimation methods were preferred because they do not require large samples to obtain useful intervals on the estimated log ORs, unlike maximum likelihood. Furthermore, we selected skeptical priors such that the Bayesian models will only give weight to the information in the data if it is very convincing, so that risk factors would only be found significant if the evidence was very strong. This approach seemed prudent given how few papers address some risk factors. Sensitivity analyses were also performed using fixed effects models.

Another limitation is that results could be affected by subgroup differences. Due to small number of papers, we could not always conduct analyses on differences between subgroups for each risk factor. Also, this study focused only on the clinical risk factors for melanoma as shown by the meta-analyses conducted by Gandini et al. (2-4). Histopathological risk factors (e.g. site, subtype, etc.) falls outside the scope of this review. Papers without a risk factor besides age and gender were not included in our analysis, so a comprehensive meta-analysis of these patient characteristics was not conducted. However, we performed analyses on these two factors with the studies that were included and which contained sufficient data. Results on age of first diagnosis based on 3 studies were inconsistent and non-interpretable (results not shown). Concerning gender, 7 studies contained enough data in the articles. A random effects model reports an OR of 0.6 (95\% HPD 0.1-3.8), indicating that gender is not significant. In addition to this result, we note that one study, Sigg et al. (19), reported that gender was not significant in their sample, which is consistent with our more general findings, but sufficient details were not provided and their study was therefore not utilized in the analysis.

Overall, this meta-analysis underlines the need for surveillance for the development of more primary melanomas in already diagnosed melanoma patients with at least one dysplastic naevus and positive family history. Light hair color seems to indicate that fair features might also be associated with multiple melanoma development but other fair phenotype markers failed to show significance, which might be due to the limited amount of papers and presence of heterogeneity. The same problem applies to sun exposure and actinic damage indicators where no significant outcomes were found in the Bayesian approach. Clearly, there is a need for additional studies regarding this subject.

\section{Acknowledgements}

Vivien Marasigan and Eric Schmitt contributed approximately equally to this work and share first-authorship.

\section{References}

1. Holterhues C, Vries E, Louwman MW, Koljenović S, Nijsten T. Incidence and trends of cutaneous malignancies in the Netherlands, 1989-2005. J Invest Dermatol. 2010;130:1807-12. Epub 2010 Mar 25.

2. Gandini S, Sera F, Cattaruzza MS, Pasquini P, Abeni $D$, Boyle P, Melchi. Meta-analysis of risk factors for cutaneous melanoma: I. Common and atypical naevi. Eur J Cancer. 2005;41:28-44.

3. Gandini S, Sera F, Cattaruzza MS, Pasquini P, Abeni $D$, Boyle P, Melchi. Meta-analysis of risk factors for cutaneous melanoma: II. Sun exposure. Eur J Cancer. 2005;41:45-60.

4. Gandini S, Sera F, Cattaruzza MS, Pasquini P, Abeni D, Boyle P, Melchi. Meta-analysis of risk factors for cutaneous melanoma: III. Family history, actinic damage and phenotypic factors. Eur $\mathrm{J}$ Cancer. 2005;41:2040-59.

5. Ferrone CR, Ben Porat L, Panageas KS, Berwick M, Halpern AC, Patel A, Coit DG. Clinicopathological features of and risk factors for multiple primary melanomas. JAMA. 2005;294:1647-54.

6. Vecchiato A, Pasquali S, Menin C, Montesco MC, Alaibac M, Mocellin S, Campana LG, Nitti D, Rossi CR. Histopathological characteristics of subsequent melanomas in patients with multiple primary melanomas. J Eur Acad Dermatol Venereol. 2012;28:58-64.

7. Siskind V, Hughes MC, Palmer JM, Symmons JM, Aitken JF, Martin NG, Hayward NK, Whiteman DC. $\mathrm{Nevi}$, family history, and fair skin increase the risk of second primary melanoma. J Invest Dermatol. 2011;131:461-7.

8. Begg CB1, Hummer AJ, Mujumdar U, Armstrong BK, Kricker A, Marrett LD, Millikan RC, Gruber SB, Culver HA, Zanetti R, Gallagher RP, Dwyer T, Rebbeck TR, Busam K, From L, Berwick M; GEM Study Group. A design for cancer case-control studies using only incident cases: experience with the GEM study of melanoma. Int J Epidemiol. 2006;35:756-64.

9. Stroup DF, Berlin JA, Morton SC, et al. Meta-analysis of Observational Studies in Epidemiology: a proposal for reporting. JAMA. 2000;283:2008-2012.

10. Lunn D, Spiegelhalter D, Thomas A, et al. The BUGS project: Evolution, critique, and future directions. Stat Med. 2009;28:3049-3067.

11. Wijeysundera DN, Austin PC, Hux JE, Beattie WS, Laupacis A. Bayesian statistical inference enhances the interpretation of contemporary randomized controlled trials. J Clin Epidemiol. 2009;62:13-21.

12. Viechtbauer W. Conducting meta-analyses in $\mathrm{R}$ with the metafor package. J Stat Soft. 2010;36:1-48.

13. Marghoob AA, Slade J, Kopf AW, Salopek TG, Rigel DS, Bart RS. Risk of developing multiple primary cutaneous melanomas in patients with the classic atypical-mole syndrome: a case-control study. $\mathrm{Br} \mathrm{J}$ Dermatol. 1996;135:704-11.

14. Scheibner A, Milton GW, McCarthy WH, Norlund JJ, Pearson LJ. Multiple primary melanoma - a review of 90 cases. Australas J Dermatol. 1982;23:1-8.

15. de Giorgi V, Rossari S, Papi F, Gori A, Alfaioli B, Grazzini M, Crocetti E, Verdelli A, Foo CW, Lotti T. Multiple 
primary melanoma: the impact of atypical naevi and follow up. Br J Dermatol. 2010;163:1319-22.

16. Hwa C, Price LS, Belitskaya-Levy, et al. Single versus multiple primary melanomas: old questions and new answers. Cancer. 2012;118:4184-92.

17. Pastorino L, Bonelli L, Ghiorzo P, Queirolo P, Battistuzzi L, Balleari E, Nasti S, Gargiulo S, Gliori S, Savoia $P$, Abate Osella $S$, Bernengo MG, Bianchi Scarrà G. CDKN2A mutations and MC1R variants in Italian patients with single or multiple primary melanoma. Pigment Cell Melanoma Res. 2008;21(6):700-9.

18. Burden AD1, Newell J, Andrew N, Kavanagh G, Connor JM, MacKie RM. Genetic and environmental influences in the development of multiple primary melanoma. Arch Dermatol. 1999;135:261-5.

19. Sigg C, Pelloni F, Schnyder UW. Increased incidence of multiple melanoma in sporadic and familial dysplastic nevus cell syndrome. Hautarzt. 1989;40: 548-52.

20. Manganoni AM1, Pavoni L, Farisoglio C, Sereni E, Chiudinelli M, Calzavara-Pinton P. Association between multiple cutaneous melanoma and other primary neoplasms. Clin Exp Dermatol. 2012;37:857-61.

21. Nagore E, Botella-Estrada R, Garcia-Casado Z, et al. Comparison between familial and sporadic cutaneous melanoma in Valencia, Spain. J Eur Acad Dermatol Venereol. 2008;22:931-6.

22. Slingluff CL Jr, Vollmer RT, Seigler HF. Multiple primary melanoma: incidence and risk factors in 283 patients. Surgery. 1993;113:330-9.

23. Titus-Ernstoff L, Perry AE, Spencer SK, et al. Multiple primary melanoma: two-year results from a population-based study. Arch Dermatol. 2006;142:433-8.
24. Titus-Ernstoff L, Duray PH, Ernstoff MS, Barnhill RL, Horn PL, Kirkwood JM. Dysplastic nevi in association with multiple primary melanoma. Cancer Res. 1988;48:1016-8.

25. Mandelcorn-Monson R, Marrett L, Kricker A, et al. Sun exposure, vitamin D receptor polymorphisms Fokl and Bsml and risk of multiple primary melanoma. Cancer Epidemiol. 2011;35:105-10.

26. Johnson TM, Hamilton T, Lowe L. Multiple primary melanomas. J Am Acad Dermatol. 1998;39: 422-7.

27. Kricker A, Armstrong BK, Goumas C, et al. Ambient UV, personal sun exposure and risk of multiple primary melanomas. Cancer Causes Control. 2007; 18:295-304.

28. Goldstein AM, Chan M, Harland M, et al. Features associated with germline CDKN2A mutations: a GenoMEL study of melanomaprone families from three continents. J MedGenet. 2007;44:99-106.

29. Yang XR1, Liang X, Pfeiffer RM, et al. Associations of 9 p21 variants with cutaneous malignant melanoma, nevi, and pigmentation phenotypes in melanoma-prone families with and without CDKN2A mutations. Fam Cancer. 2010;9:625-33.

30. Teerlink C1, Farnham J, Allen-Brady K, et al. A unique genome-wide association analysis in extended Utah high-risk pedigrees identifies a novel melanoma risk variant on chromosome arm 10q. Hum Genet. 2012;131:77-85.

31. Liang X1, Pfeiffer RM2, Li WQ, et al. Association of Genetic Variants in CDK6 and XRCC1 with the Risk of Dysplastic Nevi in Melanoma-Prone Families. J Invest Dermatol. 2014;134:481-7. 